\title{
Pertactin-Negative and Filamentous Hemagglutinin-Negative Bordetella pertussis, Australia, 2013-2017
}

\section{Zheng Xu, Sophie Octavia, Laurence Don Wai Luu, Michael Payne, Verlaine Timms, Chin Yen Tay, Anthony D. Keil, Vitali Sintchenko, Nicole Guiso, Ruiting Lan}

During the 2008-2012 pertussis epidemic in Australia, pertactin (Prn)-negative Bordetella pertussis emerged. We analyzed 78 isolates from the 2013-2017 epidemic and documented continued expansion of Prn-negative ptxP3 B. pertussis strains. We also detected a filamentous hemagglutinin-negative and Prn-negative B. pertussis isolate.

$\mathrm{D}$ espite high vaccination coverage, pertussis remains a major public health concern. In many industrialized countries, including Australia, whole-cell vaccine was replaced by the less reactogenic acellular vaccine (ACV). In Australia, the 3-component ACV (containing pertactin [Prn], pertussis toxin [Ptx], and filamentous hemagglutinin [Fha]) has been more widely used than the 5-component ACV (which also contains fimbrial antigen: Fim2 and Fim3).

Since 1991, when notifications began, pertussis has reemerged in Australia, and epidemics occur every 3-5 years. The largest epidemic occurred in 2008-2012; 39,000 cases were recorded at its peak in $2011(1,2)$. Most Bordetella pertussis isolates from that epidemic belonged to 1 genetic group, referred to as single-nucleotide polymorphism (SNP) cluster I (1-3). SNP cluster I had prn2 allele of the prn gene and $p t x P 3$ allele of the Ptx promoter (3).

In a study of the 2008-2012 epidemic, Lam et al. (1) reported a rapid increase in the number of isolates not expressing the ACV antigen Prn (Prn-negative), from 5.13\% in 2008 to $77.78 \%$ in 2012. Sequencing of 22 isolates revealed 5 epidemic lineages (ELs) (EL1-EL5) and independent

University of New South Wales, Sydney, New South Wales,

Australia (Z. Xu, S. Octavia, L.D.W. Luu, M. Payne, R. Lan); New South Wales Health Pathology and Westmead Hospital, Sydney (V. Timms, V. Sintchenko); The University of Sydney, Sydney (V. Timms, V. Sintchenko); University of Western Australia, Perth, Western Australia, Australia (C.Y. Tay); Perth Children's Hospital, Perth (A.D. Keil); Institut Pasteur, Paris, France (N. Guiso)

DOI: https://doi.org/10.3201/eid2505.180240 origins of Prn-negative strains in different ELs (4). A smaller epidemic occurred during 2013-2017, peaking at 22,000 cases in 2015 (Figure 1, panel A). We investigated the genotypic and phenotypic characteristics of $78 \mathrm{~B}$. pertussis isolates from 2013-2017 to determine the epidemic trends of pertussis in Australia.

\section{The Study}

We sequenced 78 B. pertussis isolates (Appendix 1 Table 1) from New South Wales (NSW) (17/78 [21.8\%]) and Western Australia (WA) (61/78 [78.2\%]) that were collected during the 2013-2017 epidemic. We conducted SNP detection (Appendix 1 Table 2) and examined variation in ACV antigen genes ( $p r n, p t x A, p t x P$, and the 2 fimbrial genes fim 2 and fim 3 ). Using the SNP-based classification scheme by Octavia et al. (3), we typed the 78 isolates into 2 SNP profiles (SPs): SP13 (SNP cluster I, ptxP3, 75/78 [96.2\%]) and SP18 (noncluster I, ptxP1, 3/78 [3.8\%]). All isolates harbored the ptxAl allele. Most (75/78 [96.2\%]) of the SP13 isolates had the prn 2 and fim $3 A$ alleles. The 3 noncluster I SP18 isolates had a fim $3 A^{*}$ allele that differs from fim $3 A$ by a synonymous mutation (3) with genotype ptxP1-fim $3 A^{*}$-prn1. The frequency of ptxP3 and fim $3 A$ alleles was higher than during the 2008-2012 epidemic (Figure 1, panels B, C). All but 1 isolate carried the fim 2-1 allele. One isolate (L2263 [SP18]) contained a fim2 allele with a new 3-nucleotide insertion (AGA) at position 506, resulting in the insertion of a lysine in the epitope (F2.9) region of Fim2 (5). PROVEAN analysis (6) suggests that the insertion does not affect protein structure and thus might or might not affect immune recognition. We designated this allele as fim2-3 (GenBank accession no. MG824989). Western immunoblotting showed that all isolates expressed Ptx, and all but 1 (L2228) expressed Fha. For Prn, 89.7\% (70/78) isolates were Prn-negative (Figure 1, panel D), suggesting continued expansion of Prn-negative strains.

We found multiple mechanisms of prn inactivation in the isolates, all but 1 of which were reported previously $(1,7-9)$. For most (66/70) isolates, inactivation was caused by insertion sequences (IS), including 45 IS481F insertions ( $\mathrm{F} / \mathrm{R}$ denotes insertion orientation relative to $\mathrm{prn}), 17$ IS481R insertions, and 4 IS1002R insertions (Table). We found an IS $481 \mathrm{~F}$ insertion, which has been reported in prn 1 
Figure 1. Pertussis trends and temporal changes of allele frequencies of vaccine antigens genes, Australia.

A) Pertussis notifications in Australia, 1991-2017. Source: National Notifiable Diseases Surveillance System (http://www9.health.gov.au/ cda/source/cda-index.cfm). Incidence is number of cases per 100,000 population. Reporting system was established in 1991. ACV was first introduced in Australia in 1997, and WCV was replaced with ACV in 1999. A large pertussis epidemic occurred during 2008-2012, and another epidemic occurred during 2013-2017. B) Percentage of Bordetella pertussis isolates carrying $p t x P 3$ allele in the 2 epidemic periods. C) Percentage of $B$. pertussis isolates carrying fim $3 A$ and fim $3 B$ allele in the 2 epidemic periods. D) Percentage of pertactin-expressing (Prn-positive) and pertactin-deficient (Prn-negative) B. pertussis isolates in the 2 epidemic periods. ACV, acellular vaccine; Prn, pertactin; WCV, whole-cell vaccine.

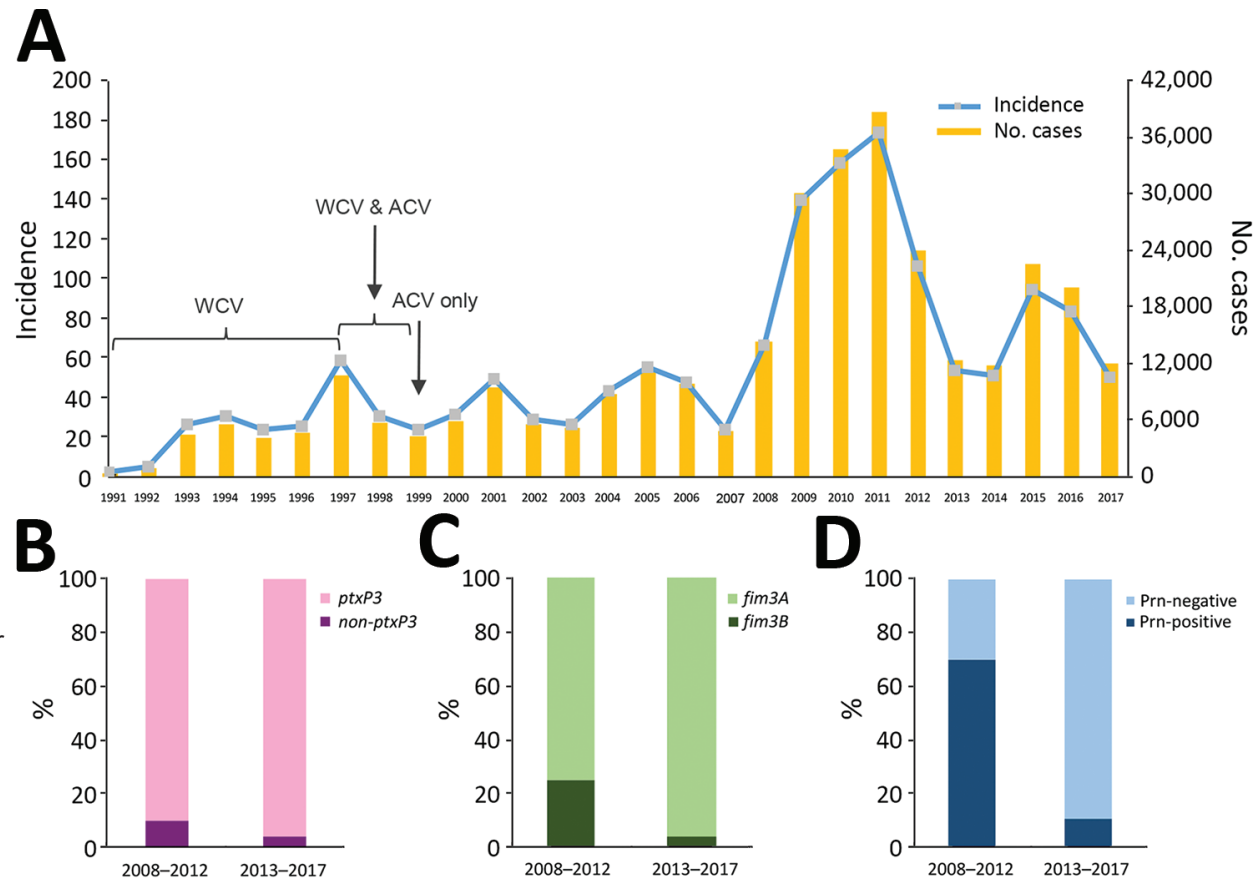

and prn 2 isolates only $(1,8)$, in 3 of the prn 3 isolates. One Prn-negative isolate contained a SNP $(\mathrm{C} \rightarrow \mathrm{T})$ in position 223 , resulting in a stop codon, a mutation found previously in US isolates only (10). Two isolates had a deletion (position $-297,1325$ [relative to the initiation codon ATG]) between the promoter and $5^{\prime}$ end of prn that was replaced with a fragment of IS1663, which might have mediated the deletion (Table). A similar but slightly different deletion (position -292, 1340) was reported in US isolates (7). We identified a new inactivation by a 4-bp deletion, from position 2020 to 2023 in prn, in 1 isolate (L2210) (Appendix 1 Table 1).

\begin{tabular}{|c|c|c|c|c|c|}
\hline $\begin{array}{l}\text { Prn deficiency } \\
\text { mechanism }\end{array}$ & Position in prn† & prn allele type & State (no. of isolates) & Year (no. isolates) & References \\
\hline IS481F & 1613 & prn2 & $\begin{array}{l}\text { Western Australia (32) } \\
\text { New South Wales (10) }\end{array}$ & $\begin{array}{c}2013(13) \\
2014(5) \\
2015(11) \\
2016(9) \\
2017(4) \\
\end{array}$ & $(1)$ \\
\hline IS481R & 1613 & prn2 & $\begin{array}{l}\text { Western Australia (12) } \\
\text { New South Wales (5) }\end{array}$ & $\begin{array}{l}2013(6) \\
2014(5) \\
2015(4)\end{array}$ & (1) \\
\hline IS481F & 1598 & prn3 & Western Australia (3) & $\begin{array}{l}2013(1) \\
2014(2)\end{array}$ & This study \\
\hline IS1002R & 1613 & prn2 & Western Australia (4) & $\begin{array}{l}2013(2) \\
2016(1) \\
2017(1)\end{array}$ & (1) \\
\hline Deletion & -297 to 1325 & Not determined $\ddagger$ & Western Australia (2) & $\begin{array}{l}2014(1) \\
2015(1)\end{array}$ & $\begin{array}{l}\text { (8), newly found } \\
\text { in Australia }\end{array}$ \\
\hline Stop codon & 223 & prn2 & Western Australia (1) & $2014(1)$ & $\begin{array}{l}\text { (10), newly found } \\
\text { in Australia }\end{array}$ \\
\hline Deletion & $2020-2023$ & prn2 & Western Australia (1) & $2013(1)$ & This study \\
\hline
\end{tabular}

${ }^{*} \mathrm{~F} / \mathrm{R}$ denotes IS insertion orientation relative to prn. F, forward; IS, insertion sequence; Prn, pertactin; R, reverse.

tThe nucleotide positions are relative to the initiation codon (ATG) of the prn in Tohama I.

$\ddagger p r n$ allele type was not determinable because the repeat regions that define prn allele type were deleted in this mechanism. 
One Prn-negative isolate (L2228) was also Fha-negative (i.e., Prn-, Fha-) by Western immunoblotting. The Fha inactivation probably resulted from changes within the homopolymeric $G$ tract (site: 1078-1087) from 10 Gs to $11 \mathrm{Gs}$ in $f h a B$, resulting in a downstream stop codon that produces a truncated FhaB protein (11). Both Illumina and Sanger sequencing (Appendix 2) showed a mixture of 10 Gs and 11 Gs. The bacterial population most likely contained predominantly 11 Gs with a lower proportion of 10 Gs. Proteomic analysis using liquid chromatography tandem mass spectrometry (12) found that, in the whole cell of L2228, only $2.3 \%$ of the FhaB protein was detected as peptides and derived mainly from the first 350 aa of the FhaB protein. In contrast, in the Fha-positive isolate (L2248), $30.7 \%$ of the FhaB protein was detected as peptides and derived from the entire protein. However, in the supernatant of L2228, we detected peptides across the entire FhaB protein and at a higher coverage of $22.7 \%$ than for wholecell FhaB. For the Fha-positive isolate, we detected $52.0 \%$ of the FhaB across the entire protein. Western immunoblotting could not detect any FhaB in supernatant or whole-cell proteins of the Fha-negative isolate.

Together with the $27 \mathrm{~B}$. pertussis isolates from Australia previously sequenced, we analyzed a total of $105 \mathrm{~B}$. pertussis isolates to determine their genomic relationships (Figure 2). Five preepidemic SP13 isolates from 1997-2002 were ancestral to the SP13 epidemic clade as expected; 3 noncluster I ( $p t x P 1)$ isolates grouped together as a separate clade outside SNP cluster I. Most (68/75) isolates grouped into 4 previously defined ELs (EL1-EL4) (4). However, no isolates from the new epidemic fell into the 2008-2012 EL5. Four isolates (L2233, L2234, L2261, and L2262) did not cluster with any of the ELs.

Prn-positive isolates from the 2008-2012 and 20132017 epidemics were distributed among different lineages. Prn-negative isolates were largely grouped by mechanism of inactivation in different ELs. Prn-negative isolates in EL1 and EL4 were caused by IS481R insertion. All but 1 Prn-negative isolate in EL2 was caused by IS1002 insertion; the exception was an IS481R insertion. Prn-negative isolates in EL3 were caused by IS481F insertion. Three Prn-negative isolates with new inactivation mechanisms found in Australia were distributed in EL4 (prn::del [-297, 1325]; note that the prn allele was indeterminate) and nonELs (prn2::stop [C233T]).

EL1 contained isolates from NSW (6/20) and WA $(14 / 20)$. EL2 was a small lineage ( 8 isolates), but these isolates were from both periods and both states. EL3 was predominantly a WA lineage; 30/33 isolates from WA and nearly half of the WA isolates (30/61) from 2013-2017 were EL3. EL4 was largely an NSW lineage (14/23 isolates).

\section{Conclusions}

The 2013-2017 pertussis epidemic in Australia was predominantly caused by Prn-negative strains, with local and

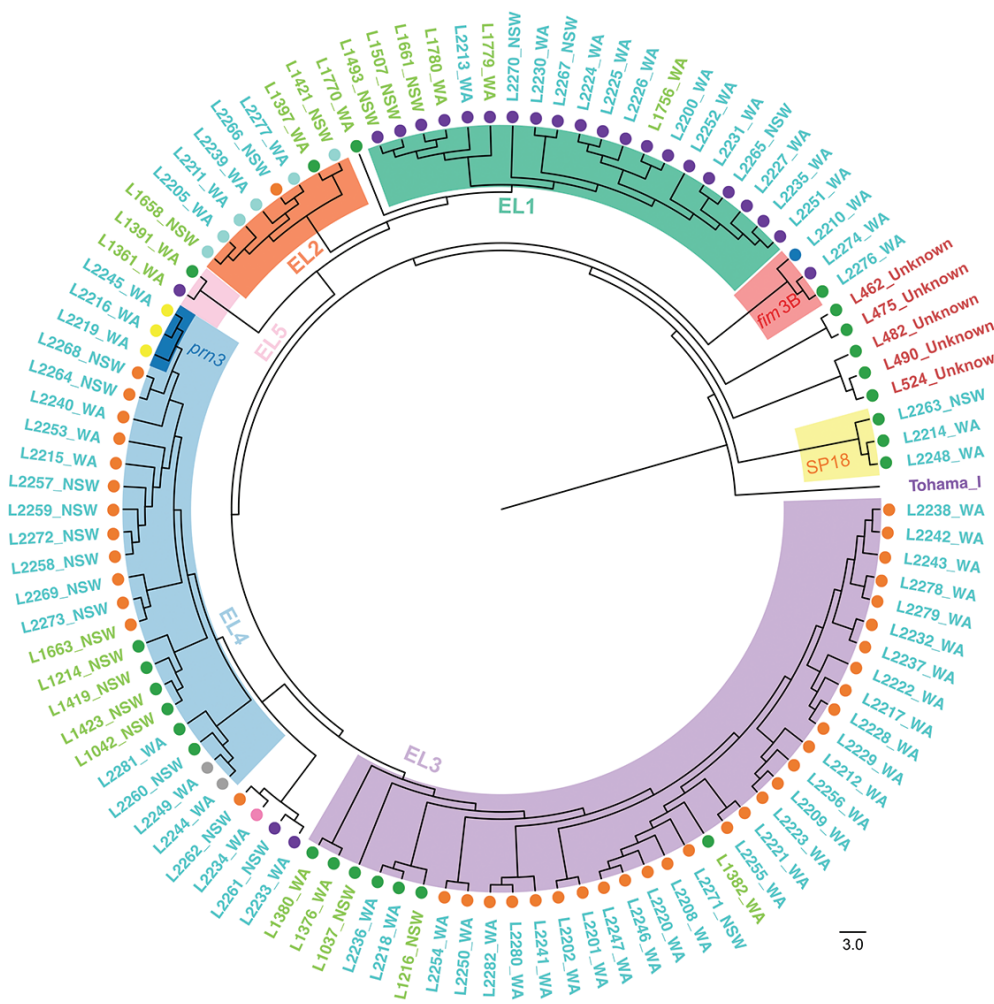

$\begin{array}{ll}\text { Location } & \text { Year } \\ \text { Unknown } & 1997-2002 \\ \text { WAINSW } & 2008-2012 \\ \text { WAINSW } & 2013-2017\end{array}$

Prn production

Prn+

Prn negative mechanisms

prn2::IS481F

prn2::IS481R

prn2::IS1002R

prn3::IS481F

prn2::stopC223T

prn2:::del $(2,020,2,023)$

prn::del $(-297$ to 1,325$)$
Figure 2. Phylogenomic relationship of 105 Bordetella pertussis epidemic isolates from Australia. The minimumevolution tree was constructed using 705 SNPs. The isolates are labeled by name (L numbers), followed by states with color to indicate years of isolates. Shaded areas inside circle indicate ELS (EL1-EL5) and branches with isolates belonging to SP18 and those carrying fim3B and prn3 alleles. Prn-positive isolates and different mechanisms of Prn-negative isolates are marked by circles with different colors. Scale bar represents 3 SNPs. EL, epidemic lineage; NSW, New South Wales; Prn, pertactin; SNP, singlenucleotide polymorphism; SP, SNP profile; WA, Western Australia. 
interstate expansion of 4 epidemic lineages. The ongoing expansion of Prn-negative strains is most likely due to continued vaccine selection pressure because Australia has been using ACVs that contain Prn since their introduction. This observation contrasts with the declining circulation of Prn-negative strains in Japan, where changes in the vaccine probably caused the decrease because 2 of the 3 vaccines used after 2012 did not contain Prn (13). The emergence of an Fha-negative and Prn-negative B. pertussis in Australia may offer higher potential to escape ACVinduced immunity.

Our results provide further evidence of B. pertussis evolution under vaccine selection. Continued surveillance of $B$. pertussis will provide a better understanding of the effect of vaccination on the evolution of the pathogen and optimize strategies to reduce the occurrence of pertussis.

\section{Acknowledgments}

We thank Narelle Raven for technical assistance.

This study was supported by a grant from the National Health and Medical Research Council of Australia (grant no.1146938). Z.X. is supported by a University of New South Wales scholarship.

\section{About the Author}

$\mathrm{Ms} . \mathrm{Xu}$ is a PhD candidate in the School of Biotechnology and Biomolecular Sciences, University of New South Wales, Sydney, NSW, Australia. Her research interests include the epidemiology and evolution of human pathogens.

\section{References}

1. Lam C, Octavia S, Ricafort L, Sintchenko V, Gilbert GL, Wood N, et al. Rapid increase in pertactin-deficient Bordetella pertussis isolates, Australia. Emerg Infect Dis. 2014;20:626-33. https://doi.org/10.3201/eid2004.131478

2. Octavia S, Sintchenko V, Gilbert GL, Lawrence A, Keil AD, Hogg G, et al. Newly emerging clones of Bordetella pertussis carrying prn 2 and $p t x P 3$ alleles implicated in Australian pertussis epidemic in 2008-2010. J Infect Dis. 2012;205:1220-4. https://doi.org/10.1093/infdis/jis178
3. Octavia S, Maharjan RP, Sintchenko V, Stevenson G, Reeves PR, Gilbert GL, et al. Insight into evolution of Bordetella pertussis from comparative genomic analysis: evidence of vaccine-driven selection. Mol Biol Evol. 2011;28:707-15. https://doi.org/10.1093/ molbev/msq245

4. Safarchi A, Octavia S, Wu SZ, Kaur S, Sintchenko V, Gilbert GL, et al. Genomic dissection of Australian Bordetella pertussis isolates from the 2008-2012 epidemic. J Infect. 2016;72:468-77. https://doi.org/10.1016/j.jinf.2016.01.005

5. Williamson P, Matthews R. Epitope mapping the Fim2 and Fim 3 proteins of Bordetella pertussis with sera from patients infected with or vaccinated against whooping cough. FEMS Immunol Med Microbiol. 1996;13:169-78.

6. Choi Y, Sims GE, Murphy S, Miller JR, Chan AP. Predicting the functional effect of amino acid substitutions and indels. PLoS One. 2012;7:e46688. https://doi.org/10.1371/journal.pone.0046688

7. Weigand MR, Peng Y, Cassiday PK, Loparev VN, Johnson T, Juieng P, et al. Complete genome sequences of Bordetella pertussis isolates with novel pertactin-deficient deletions. Genome Announc. 2017;5:e00973-17. https://doi.org/10.1128/genomeA.00973-17

8. Pawloski LC, Queenan AM, Cassiday PK, Lynch AS, Harrison MJ, Shang W, et al. Prevalence and molecular characterization of pertactindeficient Bordetella pertussis in the United States. Clin Vaccine Immunol. 2014;21:119-25. https://doi.org/10.1128/CVI.00717-13

9. Otsuka N, Han HJ, Toyoizumi-Ajisaka H, Nakamura Y, Arakawa Y, Shibayama K, et al. Prevalence and genetic characterization of pertactin-deficient Bordetella pertussis in Japan. PLoS One. 2012;7:e31985. https://doi.org/10.1371/journal.pone.0031985

10. Weigand MR, Peng Y, Loparev V, Batra D, Bowden KE, Burroughs M, et al. The history of Bordetella pertussis genome evolution includes structural rearrangement. J Bacteriol. 2017;199:e00806-16. https://doi.org/10.1128/JB.00806-16

11. Bart MJ, Harris SR, Advani A, Arakawa Y, Bottero D, Bouchez V, et al. Global population structure and evolution of Bordetella pertussis and their relationship with vaccination. MBio. 2014;5:e01074. https://doi.org/10.1128/mBio.01074-14

12. Luu LDW, Octavia S, Zhong L, Raftery M, Sintchenko V, Lan R. Characterisation of the Bordetella pertussis secretome under different media. J Proteomics. 2017;158:43-51. https://doi.org/ 10.1016/j.jprot.2017.02.010

13. Hiramatsu Y, Miyaji Y, Otsuka N, Arakawa Y, Shibayama K, Kamachi K. Significant decrease in pertactin-deficient Bordetella pertussis isolates, Japan. Emerg Infect Dis. 2017;23:699-701. https://doi.org/10.3201/eid2304.161575

Address for correspondence: Ruiting Lan, School of Biotechnology and Biomolecular Sciences, University of New South Wales, Sydney, NSW 2052, Australia; email: r.lan@unsw.edu.au 